\title{
Competing spin wave emission mechanisms revealed by time-resolved x-ray microscopy
}

\author{
Nick Träger $\odot,{ }^{1}$ Filip Lisiecki, ${ }^{2}$ Robert Lawitzki, ${ }^{3}$ Markus Weigand, ${ }^{1,4}$ Hubert Głowiński, ${ }^{2}$ Gisela Schütz, ${ }^{1}$ Guido Schmitz, ${ }^{3}$ \\ Piotr Kuświk, ${ }^{2}$ Maciej Krawczyk $\odot,{ }^{5}$ Joachim Gräfe $\odot,{ }^{1, *}$ and Paweł Gruszecki ${ }^{5,2}$ \\ ${ }^{1}$ Max Planck Institute for Intelligent Systems, Stuttgart, Germany \\ ${ }^{2}$ Institute of Molecular Physics, Polish Academy of Sciences, Poznań, Poland \\ ${ }^{3}$ Institute for Materials Science, University of Stuttgart, Stuttgart, Germany \\ ${ }^{4}$ Helmholtz-Zentrum Berlin, Berlin, Germany \\ ${ }^{5}$ Faculty of Physics, Adam Mickiewicz University, Poznań, Poland
}

(Received 30 September 2020; revised 30 November 2020; accepted 2 December 2020; published 19 January 2021)

\begin{abstract}
Spin wave emission and propagation in magnonic waveguides represent a highly promising alternative for beyond-CMOS computing. It is therefore all the more important to fully understand the underlying physics of the emission process. Here, we use time-resolved scanning transmission $\mathrm{x}$-ray microscopy to directly image the formation process of the globally excited local emission of spin waves in a permalloy waveguide at the nanoscale. Thereby, we observe spin wave emission from the corner of the waveguide as well as from a local oscillation of a domain-wall-like structure within the waveguide. Additionally, an isofrequency contour analysis is used to fully explain the origin of quasicylindrical spin wave excitation from the corner and its concurrent nonreflection and nonrefraction at the domain interface. This study is complemented by micromagnetic simulations which perfectly fit the experimental findings. Thus, we clarify the fundamental question of the emission mechanisms in magnonic waveguides which lay the basis for future magnonic operations.
\end{abstract}

DOI: 10.1103/PhysRevB.103.014430

\section{INTRODUCTION}

Spin waves in ferromagnetic wires, which can be described as a collective precession of the magnetization with a spatial phase shift, are currently of high fundamental scientific interest regarding prospective technologies at the nanoscale [1-4]. The propagation of magnons, the quanta of spin waves, provide ideal basic building blocks for future magnonic logic devices beyond CMOS properties $[1,5,6]$. To ensure reliability of prospective logic operations or data communication with magnons it is crucial to understand the fundamental phenomena of excitation mechanisms and propagation properties in spatially confined magnetic structures.

In the literature, various concepts have been proposed which allow for an efficient excitation of spin waves in nanosized magnonic structures [7-13]. In doing so, the most conventional approach is a single stripline as an antenna or a coplanar waveguide (CPW) perpendicular to the long axis of a magnonic waveguide structure [14-17]. An applied radio frequency (RF) field caused by an RF current subsequently induces spin waves. In addition, the placement of a magnonic crystal between a CPW and a thin film allows for bypassing

\footnotetext{
*graefe@is.mpg.de
}

Published by the American Physical Society under the terms of the Creative Commons Attribution 4.0 International license. Further distribution of this work must maintain attribution to the author(s) and the published article's title, journal citation, and DOI. Open access publication funded by the Max Planck Society. the limitations of the microwave antenna fabrication. Thus, spin waves that are significantly shorter than from the antenna geometry itself can be induced. This concept of spin wave excitation is known in magnonics as grating coupling $[13,18,19]$. Another promising approach has been proposed where the signal line of a CPW, which is much wider than the magnonic structure, provides a global sinusoidal RF field [9,10,20]. Thus, the coupling between the applied RF field and magnetization gradients near the edge regions within a waveguide leads to spin wave emission. This method is capable of exciting both symmetric and antisymmetric higher-order spin wave modes, which could transfer the concept of optical multiplexing to prospective magnonic devices [20]. Additionally, a big advantage of global excitation compared to an antenna is the capability of inducing ultrashort wavelengths. The wavelengths of spin wave emission using an antenna are highly limited by the geometry and the physical dimensions [21]. In contrast, global excitation allows for utilizing both gradients in the magnetization and magnetic textures at the nanoscale which are not reproducible with conventional lithography processes. Thus, ultrashort spin wave emission is only limited by the size of these textures.

Since the global excitation of spin waves in magnonic waveguides provides a promising alternative to the conventional excitation by an antenna, two main mechanisms have been claimed describing the exact origin of propagating spin waves. On the one hand, a magnonic waveguide exhibits inhomogeneities of the magnetization at its edges that are caused by the demagnetizing field and the physical confinement $[9,10]$. This results in a local shift of the resonance frequency. Thus, an RF field is capable of coupling on these 
(a) XAS image $\left(\left(\sigma^{-}+\sigma^{+}\right) / 2\right)$

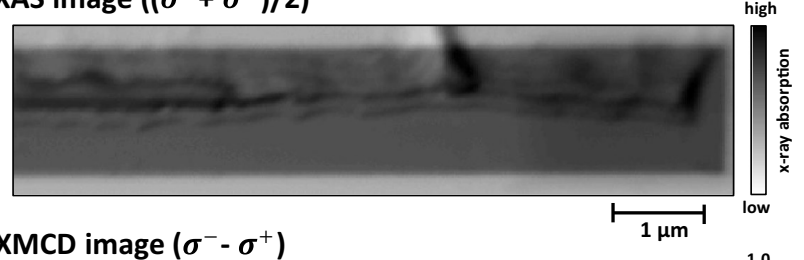

(b) XMCD image $\left(\sigma^{-}-\sigma^{+}\right)$

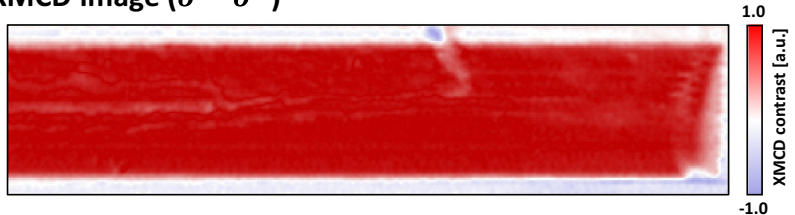

(c) TEM image

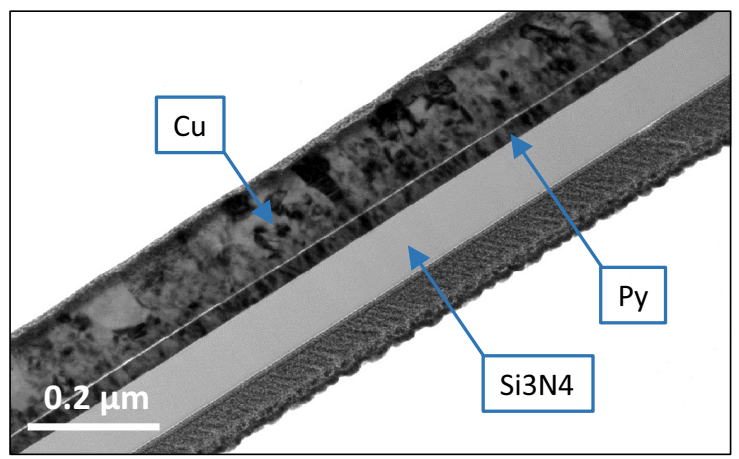

FIG. 1. (a) XAS image of a magnonic Py waveguide. (b) XMCD image of the magnonic Py waveguide shown in (a). It demonstrates a homogeneous distribution of the XMCD contrast which excludes the existence of ripple domains. (c) Bright-field TEM cross section image of the waveguide sample. Besides the silicon nitride $\left(\mathrm{Si}_{3} \mathrm{Ni}_{4}\right)$ and the copper $(\mathrm{Cu})$ of the $\mathrm{CPW}$, the cross section of the Py waveguide is visible which shows no formation of significant grain structures with sufficient size which could also lead to an excitation.

variations in the magnetization which subsequently leads to spin wave emission. On the other hand, domain wall structures in magnonic systems can act as local oscillators driven by a global RF field which results in spin wave emission [22-24]. Additionally, the existence of ripple domains in a polycrystalline waveguide has been reported which could cause an additional emission effect [11]. However, we excluded this effect in this study by magnetic $\mathrm{x}$-ray circular dichroism (XMCD) and transmission electron microscopy (TEM) measurements where no domains or sufficient grain sizes are visible (cf. Fig. 1).

In this work, we clarify the question of which mechanisms are involved in the globally excited emission of spin waves in magnonic waveguides by time-resolved scanning transmission X-ray microscopy (STXM). To this end, a magnonic permalloy waveguide (Py, $\mathrm{Fe}_{20} \mathrm{Ni}_{80}$ ) below the signal line of a CPW as shown schematically in Fig. 2(a) is excited by an oscillating sinusoidal RF current $I_{\mathrm{RF}}$. This leads to a global RF field which induces spin wave emission. To identify the exact origin, we initially perform continuous wave $(\mathrm{CW})$ excitation measurements to reveal the underlying emission mechanisms and to image them selectively. Furthermore, burst measurements are performed revealing the precise starting process of excited spin waves. For that purpose, timeresolved STXM with XMCD provides the necessary spatial

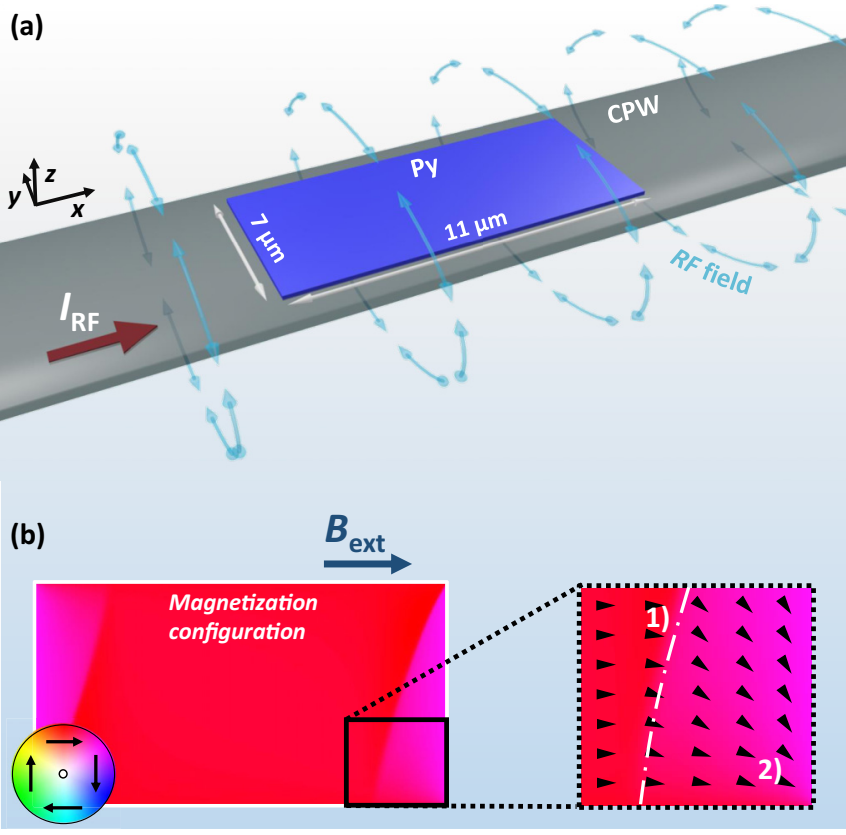

FIG. 2. (a) Schematic illustration of the Py waveguide (blue) below a CPW (gray) with the shown dimensions. The blue arrows represents the oscillating global RF field which is induced by the RF current $I_{\mathrm{RF}}$ (red arrow). (b) Micromagnetic simulation of the relaxed magnetization configuration of the Py waveguide. The color code shows the in-plane direction of the magnetization. The zoomed inset shows the area of interest where (1) a domain-wall-like structure and (2) a strong gradient in the magnetization direction can be observed. The black arrows indicate the magnetization direction. This area is used for further experiments and simulations in Figs. 3 and 5.

resolution $(<20 \mathrm{~nm})$ which exceeds the capabilities of conventional methods like Brillouin light scattering (BLS) or time-resolved scanning Kerr microscopy (TRSKM) $[8,25,26]$. Additionally, phase, amplitude, and $k$-space information are directly accessible. This study is complemented by micromagnetic simulations which perfectly fit the experimental findings. Moreover, we use an isofrequency contour (IFC) analysis to fully determine the origin and the behavior of quasicylindrical spin waves from the corner of the waveguide. This analysis also explains nonreflection and nonrefraction of this mode at the domain-wall-like structure. These results finally clarify the origin of spin wave excitation in magnonic waveguides providing a crucial building block of prospective magnonic devices.

\section{METHODS}

To realize an experiment where emission mechanisms in magnonic waveguides can be observed, the sample has to possess the mentioned magnetization gradients and a magnetic texture. For that reason, a magnonic Py waveguide (length $l=11 \mu \mathrm{m}$, width $w=7 \mu \mathrm{m}$, thickness $d=50 \mathrm{~nm}$ ) was deposited on a silicon nitride membrane by e-beam lithography. Subsequently, a CPW (copper) was fabricated on top of the signal line of the CPW as shown in Fig. 2(a).

Time-resolved STXM measurements were performed at the MAXYMUS end station at the UE46-PGM2 beam line 
at the BESSY II synchrotron radiation source. The sample was illuminated by circular polarized $\mathrm{x}$ rays at the $L_{3}$ edge of iron. An internal magnetic in-plane field ( $\pm 240 \mathrm{mT})$ was applied using a set of four rotatable permanent magnets [27]. Furthermore, time-resolved STXM provides a temporal and spatial resolution of $<40 \mathrm{ps}$ and $<20 \mathrm{~nm}$, respectively.

Each time-resolved STXM measurement contains a stack of real-space images with predefined temporal and spatial resolution. To gain amplitude and phase information, a fast Fourier transformation (FFT) algorithm is used through each pixel with respect to time. Thus, each slice of the transformed image stack provides phase and amplitude information at a specific frequency. Furthermore, a spatial 2D-FFT of the phase and amplitude map shows the corresponding $k$-space image. More detailed information of the evaluation process can be found elsewhere $[15,20]$.

The generation of $\mathrm{CW}$ and burst excitation signals were generated by an arbitrary waveform generator (AWG; Keysight Technologies M8195A) which allows for the creation of various excitation signals up to $f_{\text {out }} \leqslant 30 \mathrm{GHz}[26,28]$.

Micromagnetic simulations were performed using $\mathrm{Mu}-$ Max3 [29] with the saturation magnetization $M_{\mathrm{s}}=760$ $\mathrm{kA} \mathrm{m}^{-1}$, the exchange constant $A_{\mathrm{ex}}=13 \mathrm{pJ} \mathrm{m}^{-1}$, the damping constant $\alpha=0.008$, and the gyromagnetic ratio $\gamma=176$ rad $\mathrm{ns}^{-1} \mathrm{~T}^{-1}$ [30]. This system has been discretized with the unit cells of lateral dimensions equal $(15 \times 15) \mathrm{nm}^{2}$ and one unit cell across the thickness $(d=50 \mathrm{~nm})$. Moreover, the convergence of the results was verified; i.e., additional simulations made for a finer mesh gave equivalent results.

For the investigation by bright-field TEM the samples were prepared by focused ion beam (FIB) lift-out with a dual-beam FEI SCIOS FIB-SEM on top of a copper grid and subsequent thinning. The investigations were performed on a Philips CM200 FEG TEM operated at $200 \mathrm{kV}$. The TEM measurements were complemented by energy-dispersive $\mathrm{X}$-ray spectroscopy (EDX) measurements to identify the different layers of the sample.

\section{RESULTS AND DISCUSSION}

Before the presentation and the discussion of the experimental results of the present waveguide, we want to address an issue which was published by Kruglyak et al. in 2008 [11]. There, domains of quasiperiodic ripples were detected within a polycrystalline magnonic waveguide by TRSKM which were also excited by a global oscillating field. Therefore, we verified the existence of such ripple domains in the present sample composition to exclude an influence or even an falsification on the emission process. Figure 1(a) shows an $\mathrm{x}$-ray absorption spectroscopy (XAS) image of a narrower waveguide with the same manufacturing parameters as the waveguide in Fig. 2. The change in the width should enhance the visibility of potential domains. $\sigma^{-}$and $\sigma^{+}$represent the negative and positive helicity of the circularly polarized x-ray beam. The darker region presents the Py waveguide. While Fig. 1(a) shows only the nonmagnetic absorption, the image in Fig. 1(b) reveals the XMCD contrast. Besides some small defects caused by the lithography process, no ripple domains can be observed within the magnonic waveguide.
The XMCD image shows a homogeneous contrast distribution across the whole stripe. Additionally, Fig. 1(c) presents a cross section image performed by bright-field TEM which reveals the exact layer structure of the Py stripe. The different layers were identified by EDX measurements. Between the silicon nitride $\left(\mathrm{Si}_{3} \mathrm{~N}_{4}\right)$ and the copper $(\mathrm{Cu})$ of the $\mathrm{CPW}$, the cross section of the waveguide shows no grain sizes above typical domain wall thicknesses which could lead to any kind of domain formation or which could act as local emitters. Furthermore, no interdiffusion of the materials in each layer was visible. Consequently, this excludes the possibility of an additional excitation of ripple domains or sufficiently sized grain structures. Only a domain-like structure on the right edge can be seen in Fig. 1(b) which matches the relaxed magnetization configuration of the wider waveguide in Fig. 2.

In order to observe the proposed emission mechanisms which are based on the coupling of the RF field on variations of the magnetization and which are based on local oscillations of domain structures, the sample has to exhibit the underlying magnetic properties, i.e., magnetization variations at the edges and a domain-wall-like structure. Figure 2(b) presents the simulated relaxed magnetization configuration of a Py waveguide with the dimensions shown in (a). An external field $B_{\text {ext }}$ is applied in the $x$ direction with an offset of $B_{\text {off }}=1 \mathrm{mT}$ in the $y$ direction. The offset causes a broken mirror symmetry in the relaxed state with respect to the $y$ axis which thus recreates the experimental field setup where an offset is unavoidable. In the relaxed state, the system shows a strong change in the magnetization direction on the right lower corner which is highlighted in the zoomed inset. Additionally, a domainwall-like structure forms from the top to the bottom of the waveguide caused by the demagnetizing field and the physical confinement. Consequently, the enlarged right lower edge of the sample shows (1) a domain-wall-like structure (dashed white line) and (2) variations of the magnetization direction of approximately $45^{\circ}$ which provide an ideal environment to investigate the emission process.

The first part of the experiment presents the $\mathrm{CW}$ excitation at an excitation frequency of $f_{\mathrm{CW}}=4.1 \mathrm{GHz}$ while an external field of $B_{\text {ext }}=10 \mathrm{mT}$ is applied. The direction of the external field is defined along the $x$ axis which leads to the excitation of spin waves in backward-volume geometry; i.e., the wave vector is parallel to the direction of $B_{\text {ext }}$. The used excitation frequency and the external field were determined by micromagnetic simulations and within the experiment as the best combination for the visibility of spin waves from the domainlike structure and the corner region. All measurements were done within the marked area in Fig. 2(b) and thus, the size of each scan is $(2 \times 2) \mu \mathrm{m}^{2}$. Figure 3(a) shows the corresponding phase and amplitude map where the relative phase and amplitude are encoded into color and brightness, respectively. The dotted white line and the dash-dotted line represent the edge of the waveguide and the domain-wall-like interface, respectively. Clear propagating spin waves can be observed. However, the lower corner shows quasicylindrical emission while the left side of the image is governed by straight spin wave emission in the $x$ direction. Furthermore, the amplitude of the straight emission possesses $10 \%$ of the quasicylindrical spin wave amplitude which implies a more efficient excitation 
(a) Phase \& Amplitude map
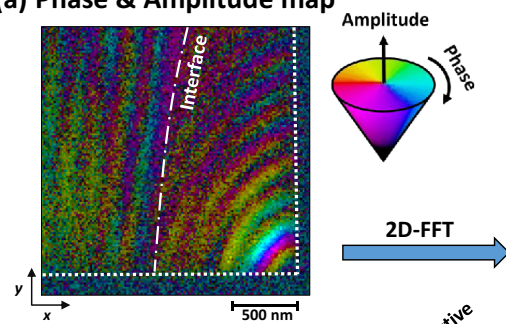

(b) $k$-space

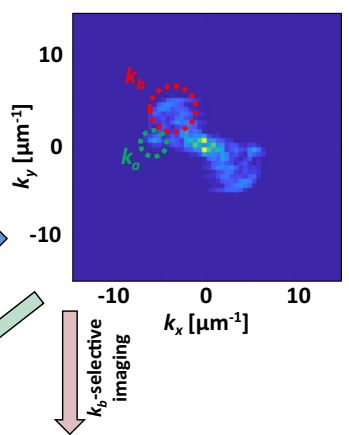

(c) $k$-selective imaging

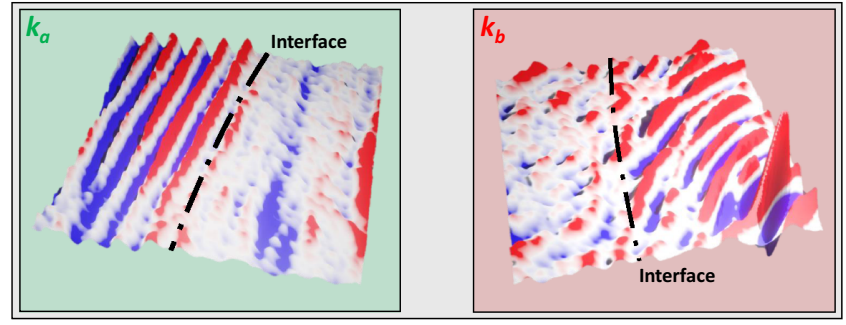

FIG. 3. (a) Phase and amplitude map of the right lower corner of the magnonic waveguide during $\mathrm{CW}$ excitation. Amplitude and relative phase are encoded into brightness and color, respectively. The image shows clear quasicylindrical spin waves from the corner and straight spin wave emission from the domain-wall-like interface (dash-dotted line). (b) $k$-space image of (a) which provides information about two distinguishable wave vectors $k_{a}$ (green) and $k_{b}$ (red). (c) Three-dimensional representation of the $k$-selective imaging of $k_{a}$ and $k_{b}$ revealing the real-space images of the corresponding wave vector.

at the corner. The origin of the quasicylindrical emission will be discussed in the latter part of this section.

To gain more information about wavelengths and propagation directions, a 2D-FFT transforms the image into $k$ space as depicted in Fig. 3(b). The $k$-space image clearly shows two significant $k$-vector areas $\left|k_{a}\right| \approx 5.7 \mu \mathrm{m}^{-1}$ and $\left|k_{b}\right| \approx$ $6.4 \mu \mathrm{m}^{-1}$ which correspond to the wavelengths $\lambda_{a} \approx 175 \mathrm{~nm}$ and $\lambda_{b} \approx 160 \mathrm{~nm}$, respectively. The quasicylindrical emission in real space is reflected in the $k$ space as a cylindrical $k$-vector distribution. The straight emission of $k_{a}$ occurs as single spot.

By emphasizing $k_{a}$ and $k_{b}$ separately, one is able to selectively image the excited spin waves as visible in Fig. 3(c) [20]. A three-dimensional representation of $k_{a}$ on the left side and $k_{b}$ on the right side substantiate the experimental results which indicate that two different origins of spin waves have to be taken into account in globally excited waveguide systems. However, CW excitation only shows the magnonic waveguide in a steady state while an excitation frequency $f_{\mathrm{CW}}$ is applied. Thus, the exact starting point of each mechanism cannot be determined. Furthermore, it is not observable if one spin wave origin affects the other. Figure 3(a) shows an abrupt change from the quasicylindrical wave to the straight emission. It might be the case that the quasicylindrical waves propagate toward the domain-wall-like structure and subsequently stimulate straight spin wave emission; i.e., they are gradually bent and refracted at the magnetization nonuniformity [31,32]. The exact emission process can be more accurately de- (a)

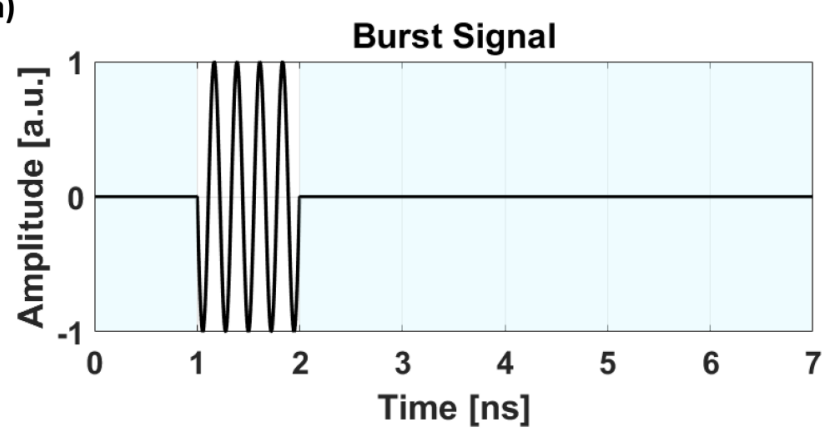

(b)

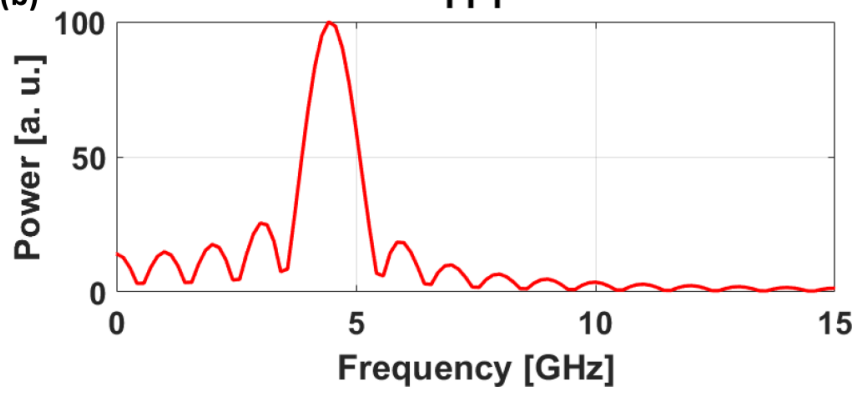

FIG. 4. (a) Illustration of a burst signal with the total length of $l=7 \mathrm{~ns}$, a burst length of $l_{\text {burst }}=1 \mathrm{~ns}$, and the carrier frequency $f_{\text {burst }}=4.2 \mathrm{GHz}$. (b) FFT spectrum of the time signal in (a).

termined by the imaging during the beginning of the excitation.

Therefore, a burst signal was generated by the AWG to investigate the starting process of the emission. Figure 4(a) shows the time signal with a total length of $l=7 \mathrm{~ns}$, a carrier frequency $f_{\text {burst }}=4.2 \mathrm{GHz}$, and a burst length $l_{\text {burst }}=1 \mathrm{~ns}$. Figure 4(b) illustrates the corresponding frequency spectrum. With this burst signal, spin waves in the range of $4 \mathrm{GHz}$ and $5 \mathrm{GHz}$ are excitable. The ripples next to the main peak in the frequency spectrum are caused by $l_{\text {burst }}$ which leads to a periodicity of $1 \mathrm{GHz}$. Simulations and experiments were performed while an external field $B_{\mathrm{ext}}=10 \mathrm{mT}$ in the $x$ direction was applied.

The results of the burst excitation are presented in Fig. 5. In (a), real-space simulations (top) and time-resolved STXM measurements (bottom) of the $m_{z}$ magnetization are compared. Three different time steps $t_{1}=0 \mathrm{~ns}, t_{2}=0.29 \mathrm{~ns}$, and $t_{3}=0.57 \mathrm{~ns}$ are depicted. $t_{1}$ defines the point where the first magnonic response can be seen in the experiment and simulation. After the initiation of the first magnonic response at $t_{1}, t_{2}(0.29 \mathrm{~ns})$ shows the moment when one period of spin waves has developed which launch from the domain-walllike structure and the lower right corner simultaneously. Both the simulation and the experiment clearly demonstrate two independent and simultaneous emission processes. However, due to the signal-to-noise ratio and the low-energy input of the burst signal, the straight spin wave emission is harder to see, while the emission of the corner is clearly visible. The images at $t_{3}$ present further propagation of the spin waves of both mechanisms. The magnons from the corner and from the domain structure show two clear periods of spin waves.

To reinforce the good agreement of the real-space simulation and the time-resolved STXM measurements, Fig. 5(b) 


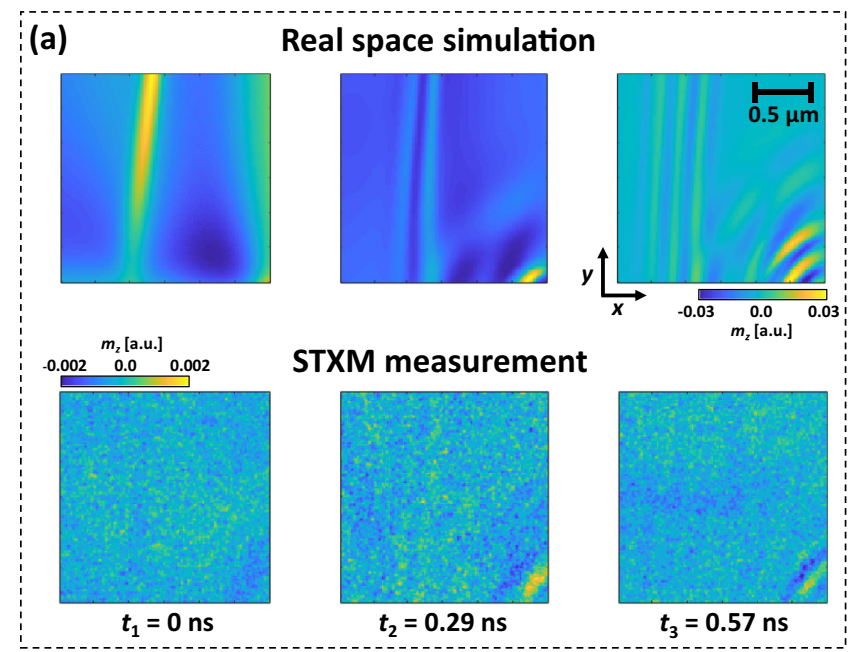

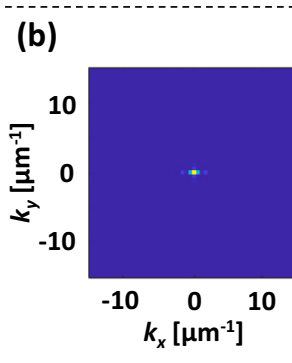
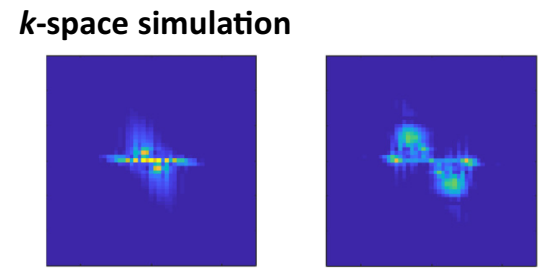

$k_{x}\left[\mu \mathrm{m}^{-1}\right]$

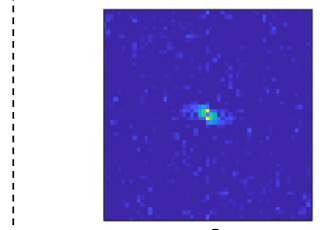

$t_{1}=0 \mathrm{~ns}$ k-space measurement

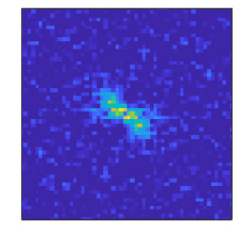

$t_{2}=0.29 \mathrm{~ns}$

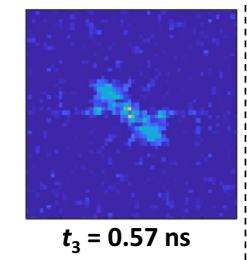

FIG. 5. (a) Demonstration of real-space simulations (top) and time-resolved STXM measurements (bottom) of the $m_{z}$ component at three different time steps $t_{1}=0 \mathrm{~ns}, t_{2}=0.29 \mathrm{~ns}$, and $t_{3}=0.57 \mathrm{~ns}$ during the burst excitation. $t_{1}$ defines the starting point of the first detected magnonic response. $t_{2}$ shows one spin wave period for each emission process. After less than 0.6 ns two spin wave periods are visible. (b) Comparison between the simulated and the measured $k$-space images. These images were calculated by a 2D-FFT of the real-space images in (a). The $k$ space reveals the simultaneous excitation of two distinguishable $k$ vectors at $t_{2}$ which reinforce the observations in (a). At $t_{3}$ the wave vectors appear more clearly because of the development of the spin wave propagation. The experimental results perfectly match the micromagnetic simulations in real space and in $k$ space.

provides the corresponding $k$-space images which were calculated by a 2D-FFT of the real-space images in Fig. 5(a). Again, one can see a perfect match of the simulation and the experimental results at all shown time steps. The initial time step $t_{1}$ shows no spin wave propagation as expected. However, two distinguishable $k$ vectors can be detected at $t_{2}$ which corresponds to the visible periods in Fig. 5(a). As already shown during CW excitation [cf. Fig. 3(b)], the diagonal $k$ vector indicates the quasicylindrical emission and the $k$ vector in $x$ direction represents the emission from the domain structure. At the last time step $t_{3}$ the $k$ vectors appear more clearly because of the multiple spin wave periods. The $k$-space evaluation also demonstrates that the two emission mechanisms start at exactly the same time and therefore independently. The time-resolved STXM measurements show excellent agreement with the micromagnetic simulation.

Subsequently, we want to address the origin of the quasicylindrical wave fronts of the spin waves propagating from the corner. The measurements and the simulations reveal a surprising result since the spin wave dynamics are supposed to be strongly anisotropic for an in-plane magnetized waveguide at low frequencies. Therefore, cylindrical-like wave fronts are not governed by the classical spin wave dispersion theory derived for uniformly in-plane magnetized thin films at low frequencies [33]. To better understand that, we have inserted two IFCs in Fig. 6(b) which represents the $k$-space image of the $m_{z}$ component in Fig. 6(a) at $t_{3}$ [31]. The first (white) IFC was calculated for the uniform film magnetized along the $x$ axis. It is visible, that the mode $k_{a}$ is located at the IFC. The second (red) IFC was calculated for a system magnetized along the axis rotated by $45^{\circ}$ with respect to the $x$ axis. This angle corresponds to the overall direction of the magnetization inside the domain near the edge [purple color in Fig. 2(b)]. The position of mode $k_{b}$ also perfectly matches the region of the rotated IFC. Moreover, the spot in Fig. 6(b) related to the mode $k_{a}$ is much narrower than the more blurred spot of $k_{b}$. It means that $k_{a}$ and $k_{b}$ correspond to a plane-wave and a beamlike excitation, respectively. A closer look at Fig. 6(a) combined with the magnetization configuration shows that the wave fronts bend together with the slight rotation of the magnetic moments [cf. Fig. 2(b)]. It is in agreement with the analytical IFC which, in an adiabatic approximation, is aligned along the magnetization direction and should rotate together with rotating magnetic moments [31].

Furthermore, the impression of cylindrical waves is amplified due to the nonalignment of the phase and group velocity (a) Real space simulation

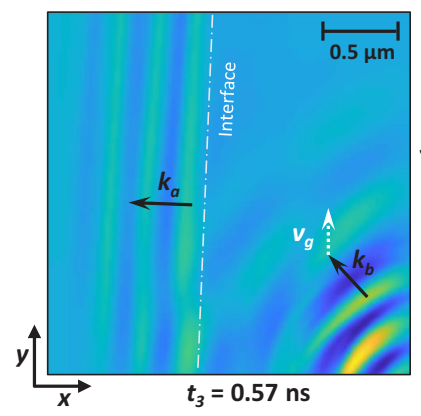

(b) $k$-space image

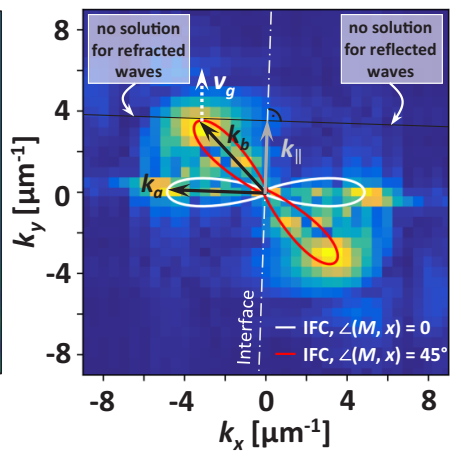

FIG. 6. (a) Snapshot of the real-space simulation at $t_{3}$. (b) $k$ space image of the snapshot in (a). The IFCs for the magnetization applied along the $x$ axis and rotated by $45^{\circ}$ with respect to the $x$ axis are marked by the white and red "peanut"-like closed curves. In (a) and (b) the dash-dotted line marks the location of the domainwall-like texture, the solid black arrows mark the directions of the wave vectors $k_{a}$ and $k_{b}$, and the dashed white arrow indicates the direction of the group velocity of the mode $k_{b} . k_{\|}$denotes the tangential component of $k_{b}$ to the interface. 
directions resulting from the anisotropy of the dispersion relation. The direction of the group velocity, $v_{g}(k)=\left[\frac{\partial \omega}{\partial k_{x}}, \frac{\partial \omega}{\partial k_{y}}\right]$ with the radial frequency $\omega$, is perpendicular to the IFC for a given wave vector $k$. Therefore, for an ideally isotropic dispersion, the IFC is circular and thus the group velocity is always parallel to $k$. However, for an anisotropic dispersion, like in the case under study, it is not true for most of the wave vectors. For $k_{b}$ the difference is equal to approximately $40^{\circ}$. In consequence, the wave fronts are tilted with respect to the $x$ axis by $45^{\circ}$, whereas the group velocity is approximately parallel to the $y$ axis as shown in Figs. 6(a) and 6(b) (dashed white arrow). As a result, we have the impression of a cylindricallike wave, although it is instead a beam propagating in the strongly anisotropic medium.

Overall, it can be said that at the very corner the backwardvolume mode is excited with the wave vector parallel to the static magnetization which follows the direction of magnetic moments. This small change of the direction of the wave fronts combined with the point-source-like excitation from the corner, the strong anisotropy of the dispersion relation, and the nonalignment of the phase and group velocity results in the appearance of quasicylindrical waves and a blurred spot for $k_{b}$ in the $k$ space.

Using the IFC analysis, we can answer the question of whether spin waves excited in the corner can propagate through the domain-wall-like structure to the central part of the waveguide or not. In order to answer this question, one has to consider Snell's law for spin waves stating that the tangential component of the wave vector to the interface must be conserved [32]. Accordingly, the tangential component of $k_{b}$ to the interface (dash-dotted line in Fig. 6) is equal to $k_{\|}$. Interestingly, there is no available solution for spin waves in the IFC of the central part of the waveguide where the magnetization is aligned along the $x$ axis. Furthermore, there are also no available solutions for reflected spin waves, i.e., $k_{x}>0$ and $k_{y}>0$. Therefore, the reflection cannot occur [34]. Overall, this observation is consistent with the burst excitation measurements (cf. Fig. 5) and the steady-state measurement shown in Fig. 3 where interference of spin waves on both sides of the interface are not visible. Notably, it is an effect similar to the total nonreflection observed experimentally for sub-millimeter-long surface magnetostatic spin waves at the line of holes $[34,35]$. However, here we have found concurrent nonreflection and nonrefraction at the magnetization nonuniformity (not holes) for ultrashort backward volume spin wave modes.

It becomes obvious that both emission mechanisms which were mentioned in the introduction have to be taken into account during the global excitation of spin waves in magnonic waveguides. Emission caused by the coupling of the RF field on magnetization gradients occurs as well as emission by the oscillation of a magnetic texture. Moreover, the results of this study demonstrate the advantage over conventional spin wave excitation by an antenna. Ultrashort spin waves below $\lambda=180 \mathrm{~nm}$ are easily excited at a comparatively low frequency of $f=4.2 \mathrm{GHz}$ which exceeds the capabilities of lithographically produced strip lines.

\section{CONCLUSION}

In conclusion, we have determined the emission mechanisms in magnonic Py waveguides which were excited by an oscillating global RF field using time-resolved STXM. In doing so, we have shown different $k$ vectors during $\mathrm{CW}$ excitation which indicated the contribution of two emission processes. To investigate the emergence of the emission, a burst signal was generated and two independent spin wave emission processes were observed. Spin waves were excited in the corner region by the coupling of the global RF field on local magnetization variations. Furthermore, a domain-wall-like structure was also excited by the RF field. The comparison between real-space and $k$-space results has shown that both emission mechanisms occur simultaneously. These STXM measurements are in perfect agreement with micromagnetic simulations. Within these simulations, we have performed IFC analyses to determine the development of the quasicylindrical spin waves which are based on a combination of a pointsource-like excitation from the corner, the nonalignment of the group and phase velocity, and the strong anisotropy of the dispersion relation. Furthermore, the IFC analysis explains the origin of the concurrent nonreflection and nonrefraction of the quasicylindrical spin wave at the domain-wall-like interface. Consequently, we have finally clarified the question of the emission process within magnonic waveguides. Thus, this study provides a fundamental basis in terms of emission schemes for prospective magnonic logic devices or data transmission in waveguides.

\section{ACKNOWLEDGMENTS}

The authors would like to thank Michael Bechtel for support during beam times. We thank HZB for the allocation of synchrotron radiation beam time. N.T., M.W., G. Schütz, and J.G. acknowledge the financial support by the Federal Ministry of Education and Research of Germany in the framework of DynaMAX (Project No. 05K18EYA). The TEM preparation was made possible by the large infrastructure support of the DFG and the Baden-Württemberg-Stiftung (INST 41/982-1). M.K. and P.G. acknowledge the financial support by the National Science Center of Poland, Grant No. UMO2018/30/Q/ST3/00416, and by the National Science Centre of Poland, Grant No. 2019/35/D/ST3/03729.
[1] A. V. Chumak, V. I. Vasyuchka, A. A. Serga, and B. Hillebrands, Magnon spintronics, Nat. Phys. 11, 453 (2015).

[2] B. Lenk, H. Ulrichs, F. Garbs, and M. Münzenberg, The building blocks of magnonics, Phys. Rep. 507, 107 (2011).
[3] A. V. Chumak, A. A. Serga, and B. Hillebrands, Magnonic crystals for data processing, J. Phys D: Appl. Phys. 50, 244001 (2017).

[4] S. Neusser and D. Grundler, Magnonics: Spin waves on the nanoscale, Adv. Mater. 21, 2927 (2009). 
[5] A. V. Chumak, A. A. Serga, and B. Hillebrands, Magnon transistor for all-magnon data processing, Nat. Commun. 5, 4700 (2014).

[6] A. Khitun, M. Bao, and K. L. Wang, Magnonic logic circuits, J. Phys D: Appl. Phys. 43, 264005 (2010).

[7] G. Dieterle, J. Förster, H. Stoll, A. S. Semisalova, S. Finizio, A. Gangwar, M. Weigand, M. Noske, M. Fähnle, I. Bykova, J. Gräfe, D. A. Bozhko, H. Y. Musiienko-Shmarova, V. Tiberkevich, A. N. Slavin, C. H. Back, J. Raabe, G. Schütz, and S. Wintz, Coherent Excitation of Heterosymmetric Spin Waves with Ultrashort Wavelengths, Phys. Rev. Lett. 122, 117202 (2019).

[8] S. Wintz, V. Tiberkevich, M. Weigand, J. Raabe, J. Lindner, A. Erbe, A. Slavin, and J. Fassbender, Magnetic vortex cores as tunable spin-wave emitters, Nat. Nanotechnol. 11, 948 (2016).

[9] F. B. Mushenok, R. Dost, C. S. Davies, D. A. Allwood, B. J. Inkson, G. Hrkac, and V. V. Kruglyak, Broadband conversion of microwaves into propagating spin waves in patterned magnetic structures, Appl. Phys. Lett. 111, 042404 (2017).

[10] C. S. Davies and V. V. Kruglyak, Generation of propagating spin waves from edges of magnetic nanostructures pumped by uniform microwave magnetic field, IEEE Trans. Magn. 52, 1 (2016).

[11] V. V. Kruglyak, P. S. Keatley, A. Neudert, M. Delchini, R. J. Hicken, J. R. Childress, and J. A. Katine, Imaging small-amplitude magnetization dynamics in a longitudinally magnetized microwire, Phys. Rev. B 77, 172407 (2008).

[12] M. Collet, O. Gladii, M. Evelt, V. Bessonov, L. Soumah, P. Bortolotti, S. O. Demokritov, Y. Henry, V. Cros, M. Bailleul, V. E. Demidov, and A. Anane, Spin-wave propagation in ultrathin YIG based waveguides, Appl. Phys. Lett. 110, 092408 (2017).

[13] C. Liu, J. Chen, T. Liu, F. Heimbach, H. Yu, Y. Xiao, J. Hu, M. Liu, H. Chang, T. Stueckler, S. Tu, Y. Zhang, Y. Zhang, P. Gao, Z. Liao, D. Yu, K. Xia, N. Lei, W. Zhao, and M. Wu, Long-distance propagation of short-wavelength spin waves, Nat. Commun. 9, 738 (2018).

[14] P. Pirro, T. Brächer, A. V. Chumak, B. Lägel, C. Dubs, O. Surzhenko, P. Görnert, B. Leven, and B. Hillebrands, Spin-wave excitation and propagation in microstructured waveguides of yttrium iron garnet/Pt bilayers, Appl. Phys. Lett. 104, 012402 (2014).

[15] F. Groß, N. Träger, J. Förster, M. Weigand, G. Schütz, and J. Gräfe, Nanoscale detection of spin wave deflection angles in permalloy, Appl. Phys. Lett. 114, 012406 (2019).

[16] F. Lisiecki, J. Rychły, P. Kuświk, H. Głowiński, J. W. Kłos, F. Groß, N. Träger, I. Bykova, M. Weigand, M. Zelent, E. J. Goering, G. Schütz, M. Krawczyk, F. Stobiecki, J. Dubowik, and J. Gräfe, Magnons in a quasicrystal: Propagation, Extinction, and Localization of Spin Waves in Fibonacci Structures, Phys. Rev. Appl. 11, 054061 (2019).

[17] P. Gruszecki, M. Kasprzak, A. E. Serebryannikov, M. Krawczyk, and W. Śmigaj, Microwave excitation of spin wave beams in thin ferromagnetic films, Sci. Rep. 6, 22367 (2016)

[18] H. Yu, G. Duerr, R. Huber, M. Bahr, T. Schwarze, F. Brandl, and D. Grundler, Omnidirectional spin-wave nanograting coupler, Nat. Commun. 4, 2702 (2013).
[19] K. Baumgaertl, J. Gräfe, P. Che, A. Mucchietto, J. Förster, N. Träger, M. Bechtel, M. Weigand, G. Schütz, and D. Grundler, Nanoimaging of ultrashort magnon emission by ferromagnetic grating couplers at $\mathrm{GHz}$ frequencies, Nano Lett. 20, 7281 (2020).

[20] N. Träger, P. Gruszecki, F. Lisiecki, F. Groß, J. Förster, M. Weigand, H. Głowiński, P. Kuświk, J. Dubowik, M. Krawczyk, and J. Gräfe, Demonstration of $k$-vector selective microscopy for nanoscale mapping of higher order spin wave modes, Nanoscale 12, 17238 (2020).

[21] V. E. Demidov, M. P. Kostylev, K. Rott, P. Krzysteczko, G. Reiss, and S. O. Demokritov, Excitation of microwaveguide modes by a stripe antenna, Appl. Phys. Lett. 95, 112509 (2009).

[22] V. Sluka, T. Schneider, R. A. Gallardo, A. Kákay, M. Weigand, T. Warnatz, R. Mattheis, A. Roldán-Molina, P. Landeros, V. Tiberkevich, A. Slavin, G. Schütz, A. Erbe, A. Deac, J. Lindner, J. Raabe, J. Fassbender, and S. Wintz, Emission and propagation of $1 \mathrm{~d}$ and $2 \mathrm{~d}$ spin waves with nanoscale wavelengths in anisotropic spin textures, Nat. Nanotechnol. 14, 328 (2019).

[23] S. J. Hermsdoerfer, H. Schultheiss, C. Rausch, S. Schäfer, B. Leven, S.-K. Kim, and B. Hillebrands, A spin-wave frequency doubler by domain wall oscillation, Appl. Phys. Lett. 94, 223510 (2009).

[24] B. Mozooni and J. McCord, Direct observation of closure domain wall mediated spin waves, Appl. Phys. Lett. 107, 042402 (2015).

[25] M. Weigand, Realization of a New Magnetic Scanning XRay Microscope and Investigation of Landau Structures under Pulsed Field Excitation (Cuvillier Verlag, Göttingen, 2015).

[26] J. Gräfe, M. Weigand, B. Van Waeyenberge, A. Gangwar, F. Groß, F. Lisiecki, J. Rychly, H. Stoll, N. Träger, J. Förster, F. Stobiecki, J. Dubowik, J. Klos, M. Krawczyk, C. H. Back, E. J. Goering, and G. Schütz, Visualizing nanoscale spin waves using MAXYMUS, Proc. SPIE 11090, 1109025 (2019).

[27] D. Nolle, M. Weigand, P. Audehm, E. Goering, U. Wiesemann, C. Wolter, E. Nolle, and G. Schutz, Note: Unique characterization possibilities in the ultra high vacuum scanning transmission x-ray microscope (UHV-STXM) "MAXYMUS" using a rotatable permanent magnetic field up to $0.22 \mathrm{~T}$, Rev. Sci. Instrum. 83, 046112 (2012).

[28] N. Träger, P. Gruszecki, F. Lisiecki, J. Förster, M. Weigand, S. Wintz, H. Stoll, H. Głowiński, P. Kuświk, M. Krawczyk, and J. Gräfe, Direct imaging of high-frequency multimode spin wave propagation in cobalt-iron waveguides using x-ray microscopy beyond 10 GHz, Phys. Status Solidi RRL 14, 2000373 (2020).

[29] A. Vansteenkiste, J. Leliaert, M. Dvornik, M. Helsen, F. GarciaSanchez, and B. Van Waeyenberge, The design and verification of MuMax3, AIP Adv. 4, 107133 (2014).

[30] T. Brächer, P. Pirro, and B. Hillebrands, Parallel pumping for magnon spintronics: Amplification and manipulation of magnon spin currents on the micron scale, Phys. Rep. 699, 1 (2017).

[31] P. Gruszecki and M. Krawczyk, Spin-wave beam propagation in ferromagnetic thin films with graded refractive index: Mirage effect and prospective applications, Phys. Rev. B 97, 094424 (2018).

[32] T. Hioki, R. Tsuboi, T. H. Johansen, Y. Hashimoto, and E. Saitoh, Snell's law for spin waves at a $90^{\circ}$ magnetic domain wall, Appl. Phys. Lett. 116, 112402 (2020). 
[33] B. A. Kalinikos and A. N. Slavin, Theory of dipole-exchange spin wave spectrum for ferromagnetic films with mixed exchange boundary conditions, J. Phys. C: Solid State Phys. 19, 7013 (1986).

[34] R. Gieniusz, P. Gruszecki, M. Krawczyk, U. Guzowska, A. Stognij, and A. Maziewski, The switching of strong spin wave beams in patterned garnet films, Sci. Rep. 7, 8771 (2017).

[35] R. Gieniusz, V. D. Bessonov, U. Guzowska, A. I. Stognii, and A. Maziewski, An antidot array as an edge for total nonreflection of spin waves in yttrium iron garnet films, Appl. Phys. Lett. 104, 082412 (2014). 